\title{
Comparison of Effect of IV Esmolol and I.V. Metoprolol For Attenuation of Pressor Response to Laryngoscopy And Intubation During Elective General Surgical Procedures Under General Anaesthesia \\ Dr Sunita Yashvant Shende Associate professor, \\ Dr Rupali N. Gorgile Assistant professor, Dr Sanyogita V. Naik Professor,
}

Dr Radhika Marathe Resident, anesthesiology, Government medical college, Miraj.

\begin{abstract}
A randomized placebo controlled single blind study was conducted to compare the effects of bolus doses of metoprolol and esmolol heart rate, systolic blood pressure and rate pressure product during laryngoscopy and intubation.60 patients of ASA I and II randomly received placebo or study group $(1 \mathrm{mg} / \mathrm{kg}$ of esmolol) or $80 \mu \mathrm{g} / \mathrm{kg}$ of metoprolol in $20 \mathrm{ml}$ normal saline. Heart rate,systolic b.p were recorded serially after study drug , before induction, during laryngoscopy and intubation and1/2min, $1 \mathrm{~min}, 11 / 2 \mathrm{~min}, 2 \mathrm{~min}$ each minute till parameters reach baseline or 20 minutes whichever is earlier. Rate pressure product was calculated and statistically evaluated in all 3 groups. Statistically significant fall in heart rate blood pressure and rate pressure product obtained in group II and III compared to control. While comparing between study drugs esmolol appears better attenuator of peak rise of blood pressure and metoprolol appears better attenuator of heart rate. Rate pressure product never reached above 15000 in any group at which myocardial ischemia may set in. Keywords: (esmolol metoprolol,laryngoscopy, intubation,rate pressure product)
\end{abstract}

\section{Introduction}

Laryngoscopy and intubation are integral parts of anaesthesiology. Sir Ivan Magills endotracheal intubation technique which he developed with Sir Horolad Gillis at Sidcup hospital, Kent was one of the most significant advances made in the field of anaesthesiology during first world war. Reid and Brane (1940) were first to recognize that cardiovascular stability may be affected by laryngoscopy and intubation. Circulatory changes are mediated by reflex sympathetic discharge due to epipharyngeal and laryngopharyngeal stimulation and are marked tachycardia, hypertension and cardiac arrhythmias. The rise in the pulse rate and blood pressure is usually transitory, unpredictable and variable. In young healthy and normotensive patients these changes are well tolerated but in patients with hypertension heart disease, coronary artery disease, the pressor response can result in increase in cardiac workload .Most of the neurosurgical patients suffer from decrease intracranial compliance due to presence of tumor or intracranial haemorrhage. Here a steep rise in blood pressure which accompanies laryngoscopy and intubation can result in rise of intracranial pressure leading to herniation of brain tissue . Over a period of time various methods are used to attenuate the pressure response .These are

- Sufficient depth of anaesthesia .

- Duration of laryngoscopy $<15$ seconds .

- Vasodilator e.g. i.v. sodium nitroprusside.

- Calcium channel blocker e.g. Nifedipine

- Lignocaine

- Beta blockers.

Beta blockers

Prys Roberts in 1971 suggested that beta blockers may attenuate the pressor response to laryngoscopy and endotracheal intubation. In 1973, he studied the effect of proctolol pressor response in hypertensive patients.He demonstrated significant attenuation pressor response along with decreased incidence of arrhythmia in patients receiving beta blockers. Magnusson $\mathbf{J}$ et al in 1986 studied pretreatment with metoprolol in hypertensive patients undergoing surgery and concluded that it was more effective than nonselective beta blocker like propranolol. In present study we have attempted to evaluate and compare the effectiveness of bolus doses of esmolol and metoprolol for attenuation of pressor response to laryngoscopy and intubation.

Aim

1) To study the hemodynamic effect of laryngoscopy and intubation in a normotensive patient using following parameters.

a) Pulse rate

b) systolic and diastolic blood pressure

c) Rate pressure product 
d) Mean arterial blood pressure

2) To compare the effectiveness of i.v. injection esmolol and metoprolol to attenuate the haemodynamic response to laryngoscopy and intubation.

\section{Material And Methods}

After obtaining permission from institutional ethics committee and written informed consent from all patients were 60 patients of either sex divided into three groups 20 patients each. Patient were randomly allocated to one of the three groups .20 patients in group one received normal saline i.v. 20 patients in group two received i.v. esmolol hydrochloride .20 patient in group three received i.v. metoprolol tartarate.

Patient of either sex in age group of 18 to 50 years undergoing different elective surgical procedures under general anaesthesia were taken up for study

- $\quad$ Patient selected were ASA group 1 and 2 patients

- Patients with predicted difficult intubations pulse less than 60 beats/min systolic blood pressure less than $100 \mathrm{~mm}$ of hg were excluded.Patient with any medical illness like D.M., Hypertension, IHD, Renal disease were excluded from the study.

- patients were investigated with all routine investigations including complete hemogram ,serum electrolytes ,random blood sugar, renal and liver function test,radiograph of chest (PA view) and ECG.

On arriving to operating room,B.P.cuff tied, pulseoximeter and ECG monitors attached. Baseline pulse, B.P.(systolic, diastolic and mean),ECG noted. I.V. cannula taken and intravenous fluid ringer's lactate started at the rate of $4 \mathrm{ml} / \mathrm{Kg} / \mathrm{hr}$. minutes.

Patients were premedicated with injection glycopyrrolate $4 \mu \mathrm{g} / \mathrm{Kg}$ and pre oxygenated with $100 \% \mathrm{O} 2$ for three

Patients in group 1 received normal saline i.v. $20 \mathrm{ml}$ bolus .Group 2 patients received injections esmolol $1 \mathrm{mg} / \mathrm{Kg}$ in 20cc normal saline. Group 3 patient received injection Metoprolol $80 \mu \mathrm{g} / \mathrm{Kg}$ in $20 \mathrm{ml}$ normal saline.

Study drugs was given three minutes prior to induction Patients were anaesthetized with inj propofol $1.5-2 \mathrm{mg} / \mathrm{kg}$ iv. Endotracheal intubation was fascilited by inj.suxamethonium $1.5 \mathrm{mg} / \mathrm{kg}$ iv. 60 seconds after inj. suxamethonium laryngoscopy and intubation was carried out by single operator who was blinded to premedication and study drug.

Non depolarizing muscle relaxant given was injection vecuronium bromide $0.1 \mathrm{mg} / \mathrm{Kg}$ intravenous after confirming the endotracheal tube position by auscultation and ET CO2 .Patient were maintained on oxygen $40 \%$ and $\mathrm{N} 2 \mathrm{O}$ $60 \%$ with IPPV on Bain's circuit .Injection Pethidine $0.5 \mathrm{mg}-1 \mathrm{mg} / \mathrm{Kg}$ and injection Phenargan $0.25-0.5 \mathrm{mg} / \mathrm{Kg}$ was given for sedation and analgesia 25 minutes after intubation .Injection diclofenac sodium $1 \mathrm{mg} / \mathrm{Kg}$ I.M., given half hour prior to the end of procedure.Following parameters were noted heart rate, systolic blood pressure ,diastolic blood pressure, ECG, $\mathrm{SpO} 2$ noted as basal awake immediately after premedication immediately after induction at laryngoscopy and intubation $1 / 2 \mathrm{~min}, 1 \mathrm{~min}, 2 \mathrm{~min}, 3 \mathrm{~min}$ each minute till parameters reach baseline or for 20 minutes whichever is earlier .Duration of laryngoscopy was noted.

Intubation score calculated based on jaw relaxation, is of laryngoscopy position of vocal cord ,coughing and limb movement .In all the groups mean and standard deviation, paired student's t test, unpaired student's t test and chi-square test were calculated using statistical package SPSS version 10-01.

III. Results

Table 1

\begin{tabular}{|l|l|l|l|l|}
\hline Metoprolol & Esmolol & Control & & \\
\hline 11 & 8 & 9 & male & Sex \\
\hline 9 & 12 & 11 & female & \\
\hline $\begin{array}{l}32.75 \pm 9.464 \\
\text { Ns }\end{array}$ & $33.70 \pm 12.04^{\mathrm{NS}}$ & $33.9 \pm 8.83$ & & Mean Age \pm SD \\
\hline $19.71 \pm 1.81^{\mathrm{Ns}}$ & $21.21 \pm 2.42^{\mathrm{Ns}}$ & $20.38 \pm 1.99$ & & BMI \\
\hline $\begin{array}{l}2.145 \pm 0.114 \\
\text { NS }\end{array}$ & $\begin{array}{l}2.105 \pm 0.105 \\
\text { Ns }\end{array}$ & $2.095 \pm 1.05$ & & Dose of propofol \\
\hline $13.90 \pm 1.210^{\mathrm{Ns}}$ & $14.00 \pm 1.77^{\mathrm{NS}}$ & $13.95 \pm 1.57$ & & Intubation Time \\
\hline
\end{tabular}

NS: Not significant

Table 2: Variations in mean heart rate in esmolol and metoprolol against control group at Various time intervals.

\begin{tabular}{|l|l|l|l|}
\hline & Control & Esmolol & Metoprolol \\
\hline Basal & $81+3.86$ & $80.1+4.02^{\mathrm{NS}}$ & $79.80+5.54^{\mathrm{NS}}$ \\
\hline $\begin{array}{l}\text { After } \\
\text { premedication }\end{array}$ & $84+4.27$ & $82.8+3.98^{\mathrm{NS}}$ & $82.3+5.24^{\mathrm{NS}}$ \\
\hline After study drug & $84+4.21$ & $77+4.02^{\text {**** }}$ & $77.6+4.96^{\text {*** }}$ \\
\hline After propofol & $84+5.77$ & $75+4.17^{\text {**** }}$ & $76.60+4.69^{\text {*** }}$ \\
\hline After scoline & $84.4+4.6$ & $76.4+4.35^{* * *}$ & $77.4+5.01^{* * *}$ \\
\hline During & $93.0+5.1$ & $79.8+3.87^{* * *}$ & $80.5+4.61^{\text {*** }}$ \\
\hline
\end{tabular}


Comparison of Effect of iv Esmolol And I.V. Metoprolol For Attenuation of Pressor Response ...

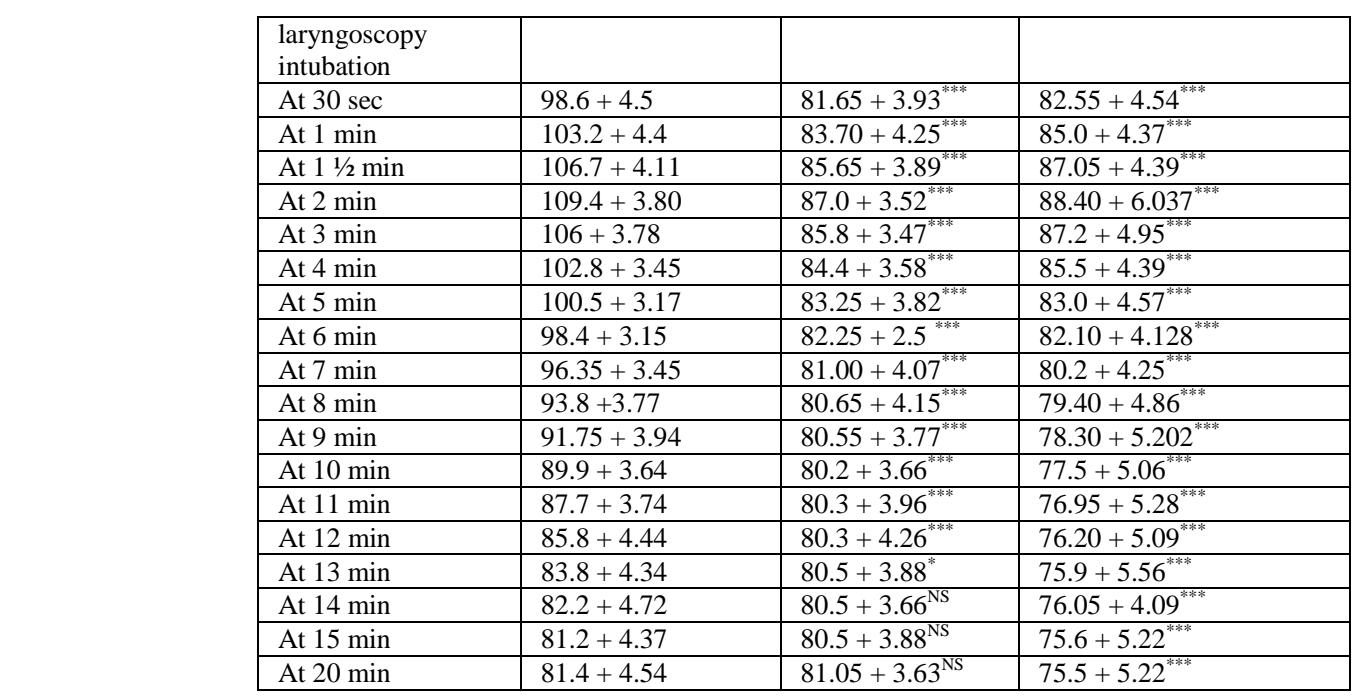

$* \mathrm{P}<0.05, * * \mathrm{P}<0.01, * * * \mathrm{P}<0.001$

NS: Not significant

Table 3: Variation in mean systolic blood pressure in esmolol and metoprolol against control group at various time intervals

\begin{tabular}{|c|c|c|c|}
\hline & Control & Esmolol & Metoprolol \\
\hline Basal & $121.7+7.84$ & $119.80+9.79^{\mathrm{NS}}$ & $118+9.613^{\mathrm{NS}}$ \\
\hline After premedication & $122.4+7.33$ & $120.7+9.52^{\mathrm{NS}}$ & $188+8.61^{\mathrm{NS}}$ \\
\hline After study drug & $122.8+7.48$ & $116.6+10.05^{*}$ & $116.3+8.21^{*}$ \\
\hline After propofol & $114.90+9.41$ & $109.7+9.782 *$ & $109.2+7.74^{*}$ \\
\hline After scoline & $116.00+6.87$ & $112.0+8.75^{*}$ & $110.9+7.152 *$ \\
\hline $\begin{array}{l}\text { At laryngoscopy \& } \\
\text { intubation }\end{array}$ & $125.8+6.89$ & $\begin{array}{l}117.7+6.937 * * \\
*\end{array}$ & $117.3+8.163^{* * *}$ \\
\hline At 30secs & $135+7.004$ & $\begin{array}{l}122.10+6.601 * \\
* *\end{array}$ & $123+7.776^{* * *}$ \\
\hline At $1 \mathrm{~min}$ & $142+7.421$ & $126+6.159$ *** & $130+7.338 * * *$ \\
\hline At $1 \frac{1}{1 / 2}$ mins & $148+8.91$ & $131.4+6.32 * * *$ & $136+5.94 * * *$ \\
\hline At 2 mins & $153.4+10.56$ & $135.6+6.57 * * *$ & $141.8+5.57$ **** \\
\hline At $3 \mathrm{mins}$ & $150.7+9.67$ & $\begin{array}{l}133.1+6.632 * * \\
*\end{array}$ & $139.2+5.709 * * *$ \\
\hline At 4mins & $145.7+9.357$ & $130.4+6.27 * * *$ & $135+5.315 * * *$ \\
\hline At $5 \mathrm{mins}$ & $141.9+9.61$ & $127.8+6.67 * * *$ & $133+4.87 * * *$ \\
\hline At $6 \mathrm{mins}$ & $138+9.42$ & $125.9+7.09 * * *$ & $131+5.44 * * *$ \\
\hline At $7 \mathrm{mins}$ & $135+9.677$ & $123.5+6.95 * * *$ & $128.5+5.18^{* * *}$ \\
\hline At $8 \mathrm{mins}$ & $132.8+9.254$ & $\begin{array}{l}122.1+7.355 * * \\
*\end{array}$ & $124.9+5.44 * * *$ \\
\hline At 9mins & $130.2+9.128$ & $\begin{array}{l}120.9+8.322 * * \\
*\end{array}$ & $121.3+6.626$ *** \\
\hline At $10 \mathrm{mins}$ & $127.70+8.56$ & $119.7+9.319^{* *}$ & $119+8.143^{* *}$ \\
\hline At $11 \mathrm{mins}$ & $126+8.182$ & $119.7+9.229 *$ & $118.2+9.40 * *$ \\
\hline At $12 \mathrm{mins}$ & $124.2+7.164$ & $120+9.798^{\mathrm{NS}}$ & $118.7+9.251^{\mathrm{NS}}$ \\
\hline Ar 13mins & $122.9+7.509$ & $119.7+9.609^{\mathrm{NS}}$ & $118.6+9.064^{\mathrm{NS}}$ \\
\hline At $14 \mathrm{mins}$ & $122.2+7.509$ & $120.1+9.27^{\mathrm{NS}}$ & $118.8+9.001^{\mathrm{NS}}$ \\
\hline At $15 \mathrm{mins}$ & $121.8+7.537$ & $120.1+9.75^{\mathrm{NS}}$ & $118.4+9.075^{\mathrm{NS}}$ \\
\hline At $20 \mathrm{mins}$ & $121.6+7.72$ & $120+9.60^{\mathrm{NS}}$ & $118+9.16^{\mathrm{NS}}$ \\
\hline
\end{tabular}

$* \mathrm{P}<0.05, * * \mathrm{P}<0.01, \quad * * * \mathrm{P}<0.001$

Table 4: variation in mean rate pressure product in esmolol and metoprolol against control groups at various time intervals:

\begin{tabular}{|l|l|l|l|}
\hline & Control & Esmolol & Metaprolol \\
\hline Basal & $9867.2 \pm 8.95$ & $9590.2 \pm 870.8^{\mathrm{NS}}$ & $9397 \pm 720.210^{\text {**** }}$ \\
\hline After premedication & $10239.3 \pm 880.25$ & $9992.4 \pm 916.66^{\mathrm{NS}}$ & $9742.6 \pm 603.412^{\text {**** }}$ \\
\hline After study drug & $10319.2 \pm 851.2$ & $8981.0 \pm 823.1^{* * *}$ & $9006 \pm 632.199^{* * *}$ \\
\hline After propofol & $9689.8 \pm 1056.5$ & $8255.1 \pm 933.4^{* * *}$ & $8351.7 \pm 623.199^{* * *}$ \\
\hline After scoline & $9797.2 \pm 856$ & $8562.7 \pm 900.9^{* * *}$ & $8577.5 \pm 633.65^{* * *}$ \\
\hline $\begin{array}{l}\text { At laryngoscopy \& } \\
\text { intubation }\end{array}$ & $11730.2 \pm 1122.8$ & $9397.9 \pm 795.837^{\text {*** }}$ & $9430.1 \pm 678.72^{* * *}$ \\
\hline
\end{tabular}


Comparison of Effect of iv Esmolol And I.V. Metoprolol For Attenuation of Pressor Response ...

\begin{tabular}{|l|l|l|l|}
\hline At 30 sces & $13349.2 \pm 1122.8$ & $9973.5 \pm 787.4^{* * * *}$ & $10192.8 \pm 706.98^{* * * *}$ \\
\hline At 1 mins & $14670.8 \pm 1231.5$ & $10598 \pm 771.75^{* * *}$ & $11080.4 \pm 656.44^{* * *}$ \\
\hline At 1 1/2 mins & $15829.4 \pm 1360$ & $11257 \pm 797^{* * *}$ & $11870.7 \pm 577.814^{* * *}$ \\
\hline At 2 mins & $16814.9 \pm 1591.7$ & $11798.8 \pm 773.23^{* * *}$ & $12534.2 \pm 969.94^{* * *}$ \\
\hline At 3 mins & $15990 \pm 1389.24$ & $11418.70 \pm 716.69^{* * *}$ & $12139 \pm 860.39^{* * *}$ \\
\hline At 4 mins & $14995 \pm 1322.1$ & $11004.8 \pm 701^{* * * *}$ & $11580 \pm 796.93^{* * *}$ \\
\hline At 5 mins & $14272.6 \pm 1224$ & $10639.3 \pm 753^{* * *}$ & $11123 \pm 796.51^{* * *}$ \\
\hline At 6 mins & $13589.0 \pm 1158.23$ & $10351.2 \pm 736.09^{* * *}$ & $10760 \pm 766.2^{* * *}$ \\
\hline At 7 mins & $13036.9 \pm 1186.73$ & $10001.4 \pm 728.96^{* * *}$ & $10307.4 \pm 704.2^{* * *}$ \\
\hline At 8 mins & $12472.6 \pm 1193$ & $9841.4 \pm 707.45^{* * *}$ & $99915.8 \pm 727.49^{* * *}$ \\
\hline At 9 mins & $11958.0 \pm 1144.03$ & $9736.2 \pm 791.28^{* * *}$ & $9486 \pm 678.26^{* *}$ \\
\hline At 10 mins & $11495.2 \pm 1089.8$ & $9601.2 \pm 891^{* * *}$ & $9204.6 \pm 658^{* * *}$ \\
\hline At 11 mins & $11064.6 \pm 1036.9$ & $9611 . \pm 883.8^{* * *}$ & $9077 \pm 774.39^{* * *}$ \\
\hline At 12 mins & $10670.80 \pm 1005.9$ & $9634.4 \pm 941.06^{* * *}$ & $9026.8 \pm 739.887^{* * *}$ \\
\hline At 13 mins & $10312.8 \pm 998.17$ & $9630 \pm 851.0^{* * * *}$ & $8982.8 \pm 763.7^{* * *}$ \\
\hline At 14 mins & $10057.4 \pm 989.62$ & $9666.8 \pm 861^{* * *}$ & $9015.6 \pm 691.87^{* * * *}$ \\
\hline At 15 mins & $9903.4 \pm 963$ & $9656.2 \pm 890^{\text {NS }}$ & $8932.8 \pm 734.02^{* * *}$ \\
\hline At 20 mins & $9910.6 \pm 965.85$ & $9763 \pm 869.39^{\text {NS }}$ & $8942.80 \pm$ \\
\hline
\end{tabular}

$* \mathrm{P}<0.05, * * \mathrm{P}<0.01, \quad * * * \mathrm{P}<0.001$

Table 5: Comparison of Heart rate at various time intervals between - Esmolol and Metoprolol group

\begin{tabular}{|c|c|c|}
\hline Time interval & Esmolol & Metoprolol \\
\hline Basal & $80.1 \pm 4.02$ & $79.80 \pm 5.54^{\mathrm{Ns}}$ \\
\hline After premedication & $82.8 \pm 3.98$ & $82.3 \pm 5.24^{\mathrm{NS}}$ \\
\hline After study drug & $77 \pm 4.02$ & $77.6 \pm 4.96^{\mathrm{Ns}}$ \\
\hline After propofol & $75 \pm 4.17$ & $76.6 \pm 4.69^{\mathrm{Ns}}$ \\
\hline After scoline & $76.4 \pm 4.35$ & $77.4 \pm 5.01^{\mathrm{Ns}}$ \\
\hline $\begin{array}{l}\text { At laryngoscopy \& } \\
\text { intubation }\end{array}$ & $79.8+3.87$ & $80.5 \pm 4.61^{\mathrm{Ns}}$ \\
\hline At 30secs. & $81.65 \pm 3.93$ & $82.5 \pm 4.54^{\mathrm{Ns}}$ \\
\hline At $1 \mathrm{mins}$ & $83.7 \pm 4.25$ & $85.0 \pm 4.37^{\mathrm{Ns}}$ \\
\hline At $1 \frac{1}{2} 2 \mathrm{mins}$ & $85.65 \pm 3.89$ & $87.0 \pm 4.39^{\mathrm{Ns}}$ \\
\hline At 2 mins & $87.0 \pm 3.52$ & $88.0 \pm 6.07^{\mathrm{Ns}}$ \\
\hline At 3 mins & $85.8 \pm 3.47$ & $87.2 \pm 4.95^{\mathrm{Ns}}$ \\
\hline At 4 mins & $84.4 \pm 3.58$ & $85.5 \pm 4.39^{\mathrm{Ns}}$ \\
\hline At 5 mins & $83.25 \pm 3.82$ & $83.6 \pm 4.57^{\mathrm{Ns}}$ \\
\hline At 6 mins & $82.25 \pm 25$ & $82.1 \pm 4.12^{\mathrm{Ns}}$ \\
\hline At 7 mins & $81.0 \pm 4.02$ & $80.2 \pm 4.25^{\mathrm{Ns}}$ \\
\hline At 8 mins & $80.5 \pm 4.15$ & $79.4 \pm 4.86^{\mathrm{Ns}}$ \\
\hline At 9 mins & $80.55 \pm 3.77$ & $78.3 \pm 5.20^{\mathrm{Ns}}$ \\
\hline At $10 \mathrm{mins}$ & $80.2 \pm 3.66$ & $77.5 \pm 5.06^{*}$ \\
\hline At $11 \mathrm{mins}$ & $80.3 \pm 3.96$ & $76.95 \pm 5.28^{*}$ \\
\hline At $12 \mathrm{mins}$ & $80.3 \pm 4.26$ & $76.2 \pm 5.09^{* * *}$ \\
\hline At 13 mins & $80.5 \pm 3.88$ & $75.95 \pm 5.56^{* * *}$ \\
\hline At $14 \mathrm{mins}$ & $80.5 \pm 3.66$ & $76.05 \pm 5.90 * *$ \\
\hline At $15 \mathrm{mins}$ & $80.5 \pm 3.88$ & $75.6 \pm 5.22 * *$ \\
\hline At $20 \mathrm{mins}$ & $81.05 \pm 3.63$ & $75.5 \pm 5.22 * *$ \\
\hline
\end{tabular}

$<0.05, * \mathrm{P}<0.01, * \mathrm{P}<0.001$

NS - Not Significant.

Table 6: Comparison of Mean systolic blood pressure at various time intervals between esmolol and metoprolol group.

\begin{tabular}{|l|l|l|}
\hline \multicolumn{1}{|c|}{ Time interval } & \multicolumn{1}{c|}{ Esmolol } & \multicolumn{1}{c|}{ Metoprolol } \\
\hline Basal & $119.80 \pm 9.79$ & $118 \pm 9.613^{\mathrm{Ns}}$ \\
\hline After premedication & $120.7 \pm 9.52$ & $118 \pm 8.61^{\mathrm{Ns}}$ \\
\hline After study drug & $116.6 \pm 10.05$ & $116 \pm 8.21^{\mathrm{Ns}}$ \\
\hline After propofol & $109.7 \pm 9.782$ & $109.2 \pm 7.74^{\mathrm{Ns}}$ \\
\hline After scoline & $112.0 \pm 8.75$ & $119.9 \pm 7.152^{\mathrm{Ns}}$ \\
\hline $\begin{array}{c}\text { At laryngoscopy \& } \\
\text { intubation }\end{array}$ & $117.7 \pm 6.937$ & $117.3 \pm 8.163^{\mathrm{Ns}}$ \\
\hline At 30secs. & $122.10 \pm 6.601$ & $123 \pm 7.776^{\mathrm{Ns}}$ \\
\hline At 1mins & $126 \pm 6.159$ & $130 \pm 7.338^{\mathrm{Ns}}$ \\
\hline At 1 ${ }^{1 / 2 m i n s}$ & $131.4 \pm 6.32$ & $136 \pm 5.54^{*}$ \\
\hline At 2mins & $135.6 \pm 6.57$ & $141.8 \pm 5.57^{*}$ \\
\hline At 3mins & $133.1 \pm 6.637$ & $139.2 \pm 5.709^{*}$ \\
\hline At 4mins & $130.4 \pm 6.27$ & $135 \pm 5.315^{*}$ \\
\hline At 5mins & $127.8 \pm 6.67$ & $133 \pm 4.87^{*}$ \\
\hline
\end{tabular}


Comparison of Effect of iv Esmolol And I.V. Metoprolol For Attenuation of Pressor Response ...

\begin{tabular}{|l|l|l|}
\hline At 6mins & $125.9 \pm 7.09$ & $131 \pm 5.44^{*}$ \\
\hline At 7mins & $123.5 \pm 6.95$ & $128.5 \pm 5.18^{*}$ \\
\hline At 8mins & $122.1 \pm 7.355$ & $124.9 \pm 5.44^{*}$ \\
\hline At 9mins & $120.9 \pm 8.322$ & $121.3 \pm 6.26^{\mathrm{Ns}}$ \\
\hline At 10mins & $119.7 \pm 9.319$ & $119 \pm 8.143^{\mathrm{Ns}}$ \\
\hline At 11mins & $119.7 \pm 9.229$ & $118.2 \pm 9.401^{\mathrm{Ns}}$ \\
\hline At. 12mins & $120 \pm 9.798$ & $118.7 \pm 9.251^{\mathrm{Ns}}$ \\
\hline At 13mins & $119.7 \pm 9.609$ & $118.6 \pm 9.064^{\mathrm{Ns}}$ \\
\hline At 14mins & $120.1 \pm 9.27$ & $118.8 \pm 9.001^{\mathrm{Ns}}$ \\
& & \\
\hline At 15mins & $120 \pm 9.75$ & $118.4 \pm 9.075 \mathrm{Ns}$ \\
\hline At 20mins & $120 \pm 9.60$ & 118 \\
\hline
\end{tabular}

$* \mathrm{P}<0.05, * * \mathrm{P}<0.01, * * * \mathrm{P}<001$

NS - Not Significant

Table 7: Comparison of Mean Rate pressure product at various time intervals between esmolol and metoprolol group:

\begin{tabular}{|l|l|l|}
\hline \multicolumn{1}{|c|}{ Time interval } & \multicolumn{1}{c|}{ Esmolol } & Metoprolol \\
\hline Basal & $9520.2 \pm 870.8$ & $9397 \pm 720.21^{\mathrm{NS}}$ \\
\hline After premedication & $9992.4 \pm 916.66$ & $9742.6 \pm 603.412^{\mathrm{Ns}}$ \\
\hline After study drug & $8981.0 \pm 823.1$ & $9006 \pm 632.199^{\mathrm{Ns}}$ \\
\hline After propofol & $8255.2 \pm 933.4$ & $8351.7 \pm 623.19^{\mathrm{Ns}}$ \\
\hline After scoline & $8562.7 \pm 900.9$ & $8577.5 \pm 633.65^{\mathrm{Ns}}$ \\
\hline At DLI & $9397.9 \pm 795.837$ & $9430.1 \pm 678.7^{* *}$ \\
\hline At 30secs. & $9973.5 \pm 787.4$ & $10192.8 \pm 706.98^{\mathrm{Ns}}$ \\
\hline At 1mins & $10598 \pm 771.75$ & $11080.4 \pm 656.4^{\mathrm{NS}}$ \\
\hline At 1 1/2 mins & $11257 \pm 797$ & $11870.7 \pm 577.81^{\mathrm{Ns}}$ \\
\hline At 2mins & $11798.8 \pm 773.23$ & $12534.2 \pm 969.9^{\mathrm{NS}}$ \\
\hline At 3mins & $11418.70 \pm 716.69$ & $12139 \pm 860.39^{\mathrm{Ns}}$ \\
\hline At 4mins & $110048 \pm 701$ & $11580 \pm 796.93^{\mathrm{Ns}}$ \\
\hline At 5mins & $10639.3 \pm 753$ & $11123 \pm 796.051^{\mathrm{Ns}}$ \\
\hline At 6mins & $10351.2 \pm 736.05$ & $10760 \pm 766.2^{\mathrm{NS}}$ \\
\hline At 7mins & $10001.4 \pm 728.96$ & $10307.4 \pm 704.2^{\mathrm{NS}}$ \\
\hline At 8mins & $9841.4 \pm 707.45$ & $9915.8 \pm 727.49^{\mathrm{Ns}}$ \\
\hline At 9mins & $9736.2 \pm 791.28$ & $9486 \pm 678.26^{\mathrm{NS}}$ \\
\hline At 10mins & $9601.2 \pm 891$ & $9204 \pm 658^{\mathrm{Ns}}$ \\
\hline At 11mins & $9611 \pm 883.8$ & $9077 \pm 774^{\mathrm{Ns}}$ \\
\hline At 12mins & $9634.4 \pm 941.06$ & $9026.8 \pm 739^{*}$ \\
\hline At 13mins & $9630 \pm 851.0$ & $8962.8 \pm 763.7^{*}$ \\
\hline At 14mins & $9666.8 \pm 861$ & $9015.6 \pm 691.8^{*}$ \\
\hline At 15mins & $9.565 \pm 890$ & $8932.8 \pm 734^{*}$ \\
\hline At 20mins & $9.763 \pm 896.39$ & $8942.8 \pm 733.2^{* *}$ \\
\hline 01 *** P 0.03 & \\
\hline
\end{tabular}

$\mathrm{P}<0.05, * * \mathrm{P}<0.01, * * * \mathrm{P}<0.001$

NS: Not significant

There was no statistically significant difference among the three groups in pre anaesthetic demographic data i.e. sex distribution ,age group, dose of propofol in milligram required for induction,intubation timeand intubation score in all three groups.

Hemodynamic trends

After study drug mean heart rate in esmolol and metoprolol has decreased over baseline heart rate and significantly lower than in control group. $(\mathrm{P}<0.001)$ while comparing metoprolol and esmolol fall in heart rate is comparable $(\mathrm{P}>0.05)$.Upto 9 minutes mean heart rate in esmolol and metoprolol group is comparable and difference is not statistically significant. After 10 minutes there is gradual decline in heart rate in metoprolol group over baseline and difference in two study groups is statistically significant for 10 minutes $(\mathrm{p}<0.05)$ and 11 minutes $(0.01)$.Mean heart rate reaches baseline at around 13 minutes after intubation in control group $(\mathrm{p}<0.05)$. But heart rate is lower in metoprolol group than control group $(\mathrm{P}<0.01)$. After inj.of study group mean systolic B.P.was significantly lower in esmolol and metoprolol group $(\mathrm{P}<0.001)$ but difference between two groups was not significant . after induction with propofol was there is much greater fall in systolic B.P. in esmolol and metoprolol group than control $(\mathrm{P}<0.001)$.

After the injection study drug and injection propofol fall in rate pressure product was much greater in esmolol and metoprolol group $(\mathrm{P}<0.001)$.During laryngoscopy and intubation rate pressure product increased in all three groups but two much greater extent in control group and maximum rise seen at two minutes after intubation which was much greater in control group than esmolol and metoprolol $(\mathrm{p}<0.001)$

RPP remain elevated over base line for about fifteen minutes in control group while in esmolo and metoprolol group it reached baseline 8 minutes and 9minutea after intubation respectively. In metoprolol group RPP remain lower than control and no difference in esmolol and metoprolol group up to 11 minutes after intubation.

\section{Discussion}

Pressor response to laryngoscopy and intubation is recognized since 1940 .The most deleterious effects of pressor response are tachycardia ,hypertension, myocardial ischemia , arrhythmias. The purpose of present study was to compare the effects of esmolol and metoprolol both cardioselective $\beta$ blockers for attenuation of pressor response to laryngoscopy and endotracheal intubation . 
Both study drugs have direct chronotropic action and cause equal fall in heart rate $(\mathrm{P}>0.005)$. Magnusson J.et al studied stress response to microlaryngoscopy with metoprololand fentanyl andhave shown thatmetoprolol causes fall in heart rate after injection $(\mathrm{p}<0.01)$. Another study by Magnusson et al in hypertensive patientsalso shown decrese in heart rate after metoprolol injection( $\mathrm{p}<0.05)$.In both studies metoprolol was given 4 days and 2 weeks prior to surgery respectively and dose used before induction intravenously was also higher 10 mgand $15 \mathrm{mg}$ iv compared to present study.Coleman et al studied $\beta$ blockade with $2 \mathrm{mg}$ and $4 \mathrm{mg}$ of metoprolol and found dose dependant fall in heart rate. Dose used by Coleman paralles dose in our study.

Greater fall in mean heart rate followingpropofol in study drugs indicates combined effect of beta blockers and propofol on heart rate.Effect of baroreceptor reflex control of heart is known with propofol.Though the pain factor on inj. propofol would be same in all three groups, sympathetic response to pain is blocked by beta antagonist esmolol and metoprolol group.Thereforepresent study shows the greater fall in heart rate in group of study drugsthan control group.Better results $(\mathrm{p}<0.001)$ obtained than Magnusson et al $(\mathrm{p}<0.01)$ indicates probable role of propofolas induction agent and additive effect on heart rate.Studies by Coleman et al ,R.Korpinen,Canadian multicenter studyby D.R.Miller and Phillip Liu shown results in accordance with present study and results are statistically better $(\mathrm{p}<0.001)$ probably related to induction agentpropofol as against thiopentone sodiuminother studies. Javed et al compared esmolol and metoprolol (25mg and 4 mgrespectively) in hypertensive patient and esmolol was found to be better in attenuating heart rate response ( $p$ $<0.001$ ).present study utilizing similar doses found no stastistically significant difference between esmolol and metoprolol. Pressor response is always appears exaggerated and unpredictable or variablein hypertensive patients.After 10 minutes heart rate in metoprolol group has decreased more compared to esmolol group $(\mathrm{p}<0.05)$. The reason for this difference in heart rate is most probably duration of action of esmolol and metoprolol .Elimination half life of esmolol is 9 minutes and action remains for $15-20$ minutes after injection whereas elimination $1 / 2$ life of metoprolol is 3-7 hours. Mechanism of attenuation of blood pressure response by beta blockers is by negative chronotropic and ionotropic action. Other mechanisms are inhibiting the release of renin,thus decreasing the angiotensin and aldosterone,decreasing central vasomotor activity.But probably these mechanisms come into play after many hours of starting drug therapy.So in study by Magnusson et althey have used oral metoprolol 4 days and 2 weeks prior to surgery and got positive results.

Rate pressure product is product of heart rate and systolic blood pressure.It is indicator of myocardial work load.Esmolol and Metoprolol have significantly decreased rate pressure product compared to control group ( $\mathrm{p}<0.001$ )which is due to negative chronotropic action of beta blockers. It is seen that mean RPP increase as soon as laryngoscopy begins and reaches peak at 2 minutes after intubation. At all time intervals RPP remains significantly higher in control group than study drugs $(\mathrm{p}<0.001)$. There was no significant difference between esmolol and metoprolol group $(\mathrm{p}>0.05)$.RPP never reached above 15000 in esmolol and metoprolol group at which myocardial ischemia sets in [ Javed et al ].

\section{Summery And Conclusion}

Based on results of present study it may be concluded that intravenous esmolol and metoprolol prevent rise in heart rate and peak rise in blood pressure with resultant rise in rate pressure product. Clinically and statistically it can be concluded that metoprolol may be a better agent for attenuation of heart rate and esmolol is better choice to attenuate blood pressure.Propofol appers to have additive effect with beta blockers on heart rate and blood pressure at lower doses of esmolol and metoprolol. No adverse effects like severe hypotensionor bradyarrythmias requiring treatment were observed in any group.

\section{Bibliography}

[1]. Magnusson J, Werner O, Carison C. et al: Metoprolol, fentanyl and stress response to microlaryngoscopy . Effect on arterial pressure, heart rate and plasma concentration of catecholamines, ACTH and cortisol .British Journal of anaesthesia 1983, 55(5);405414.

[2]. Prys - Roberts C et al: Studies of anaesthesia in relation to hypertension,adrenergic beta receptor blockade.British journal of anaesthesia.1973,45;671-680.

[3]. Magnusson J et al: Hemodynamic effects of metoprolol in hypertensive patients undergoing surgery. British J of anaesthesia 1986, 58;251-260.

[4]. Coleman AJ ,Jorden C. Cardiovascular response to anaesthesia influence of betareceptor blockade with metoprolol Anaesthesia, 1980, 35:972-978

[5]. Freysz M. Timourt Q, Betril L et al. Propofol and bradycardia. Canadian J Anaesthesia 1991; 28:137-138.

[6]. Liu P, Gatt S ,Gugino LD,Mallampatti SR,Convino BG: Esmolol for control of increase in heart rate and blood pressure during tracheal intubation after thiopentone and succinyl choline.Canadian anaesthesia society Jounrnal September1986, 33(5);556-562.

[7]. Miller R.D. Martineu R.J. Wynands J E: Bolus administration of esmolol for controlling hemodynamic response to tracheal intubation ;the Canadian multicentral trial.Canadian J Anaesthesia,1991,38(7);849-858.

[8]. Menkhaus G.P.,Reves J G,Kiesen J:Cardiovascular effects of esmolol in anaesthetized humans. Anaesthesia and analgesia1985,64;327-334.

[9]. Korpinen R. Saarnivaara L,Simola M:Effect of esmolol on heart rate ,arterial pressure and ECG changes during laryngomicroscopy;Acta Anaesthesiologica Scandinavica;1997,41:371-375.

[10]. Javed A. Zargar et al.Comparative evaluation of effect of metoprolol and esmololon rate pressure product and ECG changes during laryngoscopy and endotracheal intubation in controlled hypertensive patients. Anaesthesia 2002;46(5); 365-368 